\title{
Live Quantitative Monitoring of Mineral Deposition in Stem Cells Using Tetracycline Hydrochloride
}

\author{
Laura Macri-Pellizzeri, PhD,, ${ }^{1,2}$ Nigel De Melo, MS,, Ifty Ahmed, PhD,2 David Grant, $\mathrm{PhD}$, \\ Brigitte Scammell, $\mathrm{MD}^{3}$, and Virginie Sottile, $\mathrm{PhD}^{1}$
}

The final stage of in vitro osteogenic differentiation is characterized by the production of mineral deposits containing calcium cations and inorganic phosphates, which populate the extracellular matrix (ECM) surrounding the cell monolayer. Conventional histological techniques for the assessment of mineralization, such as Von Kossa and Alizarin Red S staining, are end point techniques requiring cell fixation. Moreover, in both cases staining quantitation requires dye extraction, which irreversibly alters the ECM conformation and structure, therefore preventing the use of the sample for further analysis. In this study, the use of tetracycline hydrochloride (TC) is proposed for the nondestructive staining, quantitation, and imaging of mineralizing bone-like nodules in live cultures of human bone marrow mesenchymal stem cells cultured under osteogenic conditions. Overnight administration of TC to living cells was shown not to alter the metabolic activity or the progression of cell differentiation. When applied to differentiating cultures, cell exposure to serial doses of TC was found to produce quantifiable fluorescence emission specifically in osteogenic cultures. Incubation with TC enabled fluorescence imaging of mineralized areas in live cultures and the combination with other fluorophores using appropriate filters. These results demonstrate that serial TC administration over the differentiation time course provides a qualitative and quantitative tool for the monitoring and evaluation of the differentiation process in live cells.

Keywords: stem cells, osteogenesis, in vitro differentiation, live cell imaging, quantitative assay

\section{Introduction}

$\mathbf{T}$ TE CEllular PROCESS underpinning bone formation is routinely modeled in vitro using different types of cells, including primary osteoblasts and multipotent and pluripotent stem cells which, under specific physicochemical stimulation, differentiate into mineralizing bone-like cells. ${ }^{1}$ During osteogenic differentiation, a well-organized and collagen-enriched extracellular matrix (ECM) is formed followed by the production of extracellular mineral deposits made of calcium and inorganic phosphates. ${ }^{2}$

In conventional 2D culture systems, these mineral deposits can be identified on top of the cell monolayer through brightfield imaging. ${ }^{3}$ However, qualitative and quantitative assessments of mineralization are essential for the full characterization of differentiation and are conventionally achieved with histological stain techniques. The most widely used assays include Von Kossa and Alizarin Red S staining, which respectively target anionic phosphates and calcium cations. ${ }^{4,5}$ However, both methods are end point assays and require cell fixation, followed by multiple staining steps. To overcome these limitations and enable live analyses, diverse fluorochrome-based labeling methods have been proposed for the evaluation of bone formation in vitro and in vivo such as Giemsa, Calcein blue, and Xylenol Orange, among others. ${ }^{6-9}$

Tetracyclines (TCs) constitute a wide family of broad spectrum antibiotics classified as natural, semisynthetic, and chemically modified according to their origin. ${ }^{10}$ In addition to

\footnotetext{
${ }^{1}$ Wolfson STEM Centre, Division of Cancer and Stem Cells, School of Medicine, The University of Nottingham, Nottingham, United Kingdom.

${ }^{2}$ Advanced Materials Group, Department of Mechanical, Materials and Manufacturing Engineering, Faculty of Engineering, The University of Nottingham, Nottingham, United Kingdom.

${ }^{3}$ Orthopaedics and Trauma Group, Division of Rheumatology, Orthopaedics, and Dermatology, School of Medicine, The University of Nottingham, Nottingham, United Kingdom.

(C) Laura Macri-Pellizzeri et al. 2018; Published by Mary Ann Liebert, Inc. This Open Access article is distributed under the terms of the Creative Commons License (http://creativecommons.org/licenses/by/4.0), which permits unrestricted use, distribution, and reproduction in any medium, provided the original work is properly cited.
} 
their antimicrobial activity, these compounds are characterized by their calcium chelating ability and fluorescence emission. ${ }^{11,12}$ These properties have led TCs to be used as a marker of calcification front in bone, applied in vivo by parenteral or enteral administration or used for staining bone biopsies postfixation. ${ }^{13}$ TCs have also more recently been used for the qualitative observation of mineralized ECM in dental pulp cell culture by fluorescence imaging. ${ }^{14}$ However, TCs have not yet been used for the quantitative evaluation of mineralization in live cultures.

The aim of this study was to investigate the use of tetracycline hydrochloride for the nondestructive in vitro staining, quantification, and live imaging of bone-like mineralized ECM using differentiating human mesenchymal stem cells (MSCs).

\section{Materials and Methods}

All reagents were purchased from ThermoFisher Scientific (UK) unless otherwise stated.

\section{Cell culture and differentiation}

Immortalized human bone marrow-derived MSCs ${ }^{15-18}$ were seeded at a density of 4000 cells $/ \mathrm{cm}^{2}$ in 48 -well plates. After $24 \mathrm{~h}$, the standard medium (SC) (low-glucose Dulbecco's modified Eagle's medium supplemented with $10 \%$ fetal calf serum, $1 \%$ penicillin and streptomycin, $1 \%$ L-Glutamine, and $1 \%$ of nonessential amino acids) was replaced with osteogenic medium (OS) (SC supplemented with $0.1 \mu \mathrm{M}$ dexamethasone, $10 \mathrm{mM} \beta$-glycerophosphate, and $50 \mu \mathrm{M}$ ascorbic acid; Sigma-Aldrich, UK) to induce osteogenic lineage differentiation. ${ }^{19}$ Cells were cultured for 21 days at $37^{\circ} \mathrm{C}$ and $5 \% \mathrm{CO}_{2}$, with medium refreshment every $48 \mathrm{~h}$.

\section{Evaluation of cytotoxicity}

Cell metabolic activity was analyzed using PrestoBlue reagent at days 7,14 , and 21 , according to the manufacturer's instructions. Briefly, the cells were washed once with phosphate buffered saline (PBS) and incubated with $300 \mu \mathrm{L}$ of SC containing $10 \%$ of PrestoBlue reagent at $37^{\circ} \mathrm{C}$ for 40 min which was within the dynamic range of the assay. Two hundred fifty microliters were transferred to a new 96well plate, and the fluorescence was measured in a microplate reader (Tecan Infinite 200) using excitation and emission wavelengths set at 560 and $590 \mathrm{~nm}$, respectively.

\section{Alkaline phosphatase assay and Alizarin Red S staining}

Alkaline phosphatase (ALP) activity was assayed at days 7 and 14 of culture. A solution containing $1 \mathrm{mg} / \mathrm{mL}$ p-nitrophenyl phosphate and $0.2 \mathrm{M}$ Tris buffer (SIGMAFAST; Sigma-Aldrich) was prepared according to the manufacturer's instructions. Cells seeded in 48-well plates were washed twice with PBS, and $300 \mu \mathrm{L}$ of assay solution was added to each well. ALP activity was monitored by performing 12 readings of the optical density at $405 \mathrm{~nm}$ over $24 \mathrm{~min}$ in a microplate reader. Cells were then washed twice, and fresh medium was added before returning the cells to the incubator until the following time point.

Alizarin Red S staining was performed at days 7, 14, or 21 as stated. Cell fixation was performed using $4 \%$ paraformaldehyde for $10 \mathrm{~min}$ at $4^{\circ} \mathrm{C}$. Before the staining, fixed cells were washed twice with deionized water. Then, $200 \mu \mathrm{L}$ of $1 \%$ w/v Alizarin Red S solution (Sigma-Aldrich) was added to each well for 10-15 min, followed by extensive washing with deionized water before imaging. For Alizarin Red S staining quantification, stained cells were washed with deionized water and incubated with $200 \mu \mathrm{L}$ of destaining solution $(20 \%$ methanol, $10 \%$ acetic acid in deionized water) during 15-20 min before measuring the absorbance of the solution at $405 \mathrm{~nm}$ in the microplate reader.

\section{Tetracycline administration and analysis of fluorescence emission}

The tetracycline staining solution chosen was prepared with tetracycline hydrochloride (TC) (Sigma-Aldrich) dissolved in PBS, filtered through $0.22 \mu \mathrm{m}$ syringe filter, and administrated to the cells at final concentrations of 5, 10, 20, or $40 \mu \mathrm{g} / \mathrm{mL}$ in culture medium at days 6,13 , and 20. After overnight incubation (18-22 h) with TC, cells were washed twice with PBS; then, $300 \mu \mathrm{L}$ of PBS was added to each well, and fluorescence was measured using the microplate reader (Tecan Infinite 200), recording 25 reading points per well at $390 \mathrm{~nm}$ excitation and $560 \mathrm{~nm}$ emission.

\section{Cell staining and imaging}

For both live and fixed samples, cell nuclei were counterstained by incubating cells with $10 \mu \mathrm{g} / \mathrm{mL}$ of Hoechst 33258 for $10 \mathrm{~min}$. Cytoskeletal actin fibers were visualized in fixed cells using VECTASHIELD mounting medium containing TRITC-Phalloidin (Vector Laboratories, UK).

Live cells were imaged on a Leica DM IRB microscope using the A filter cube (excitation filter $\lambda \quad 340-380 \mathrm{~nm}$, emission long pass filter $\lambda 425 \mathrm{~nm}$ ) and coupled to a QICAM Fast 1394 camera. Confocal laser scanning microscopy was carried out on a ZEISS Elyra PS.1 microscope equipped with LSM 780 confocal unit using $10 \times / 0.45 \mathrm{NA}$ water immersion objective. The TC emission spectrum was acquired in Lambda mode using a 32-channel meta-detector in the confocal microscope. After inspecting the emission signals, sequential imaging channels were set for each fluorophore, and for each channel the respective laser was assigned: Hoechst: $\lambda_{\text {ex. }} 405 \mathrm{~nm}$ laser, $\lambda_{\text {em. }}$ 405-437 nm; TC: $\lambda_{\text {ex. }} 405 \mathrm{~nm}$ laser, $\lambda_{\text {em. }}$ 588-650 nm; and TRITC-Phalloidin: $\lambda_{\text {ex. }} 561 \mathrm{~nm}$ laser, $\lambda_{\text {em. }} 579-641 \mathrm{~nm}$.

\section{Statistics}

Results from three independent experiments are presented as mean \pm standard error of the mean. One-way analysis of variance with Tukey's multiple comparison post hoc test was used. A 95\% confidence level was considered significant. Statistical analysis was performed with the GraphPad PRISM 7.01 software package.

\section{Results}

Tetracycline as an in vitro live stain for osteogenic differentiation

To test the ability of $\mathrm{TC}$ to stain mineralizing cells in culture and determine the optimal working concentration, differentiated and undifferentiated MSCs cultured for 20 days in vitro were incubated for $18-22 \mathrm{~h}$ with four TC 

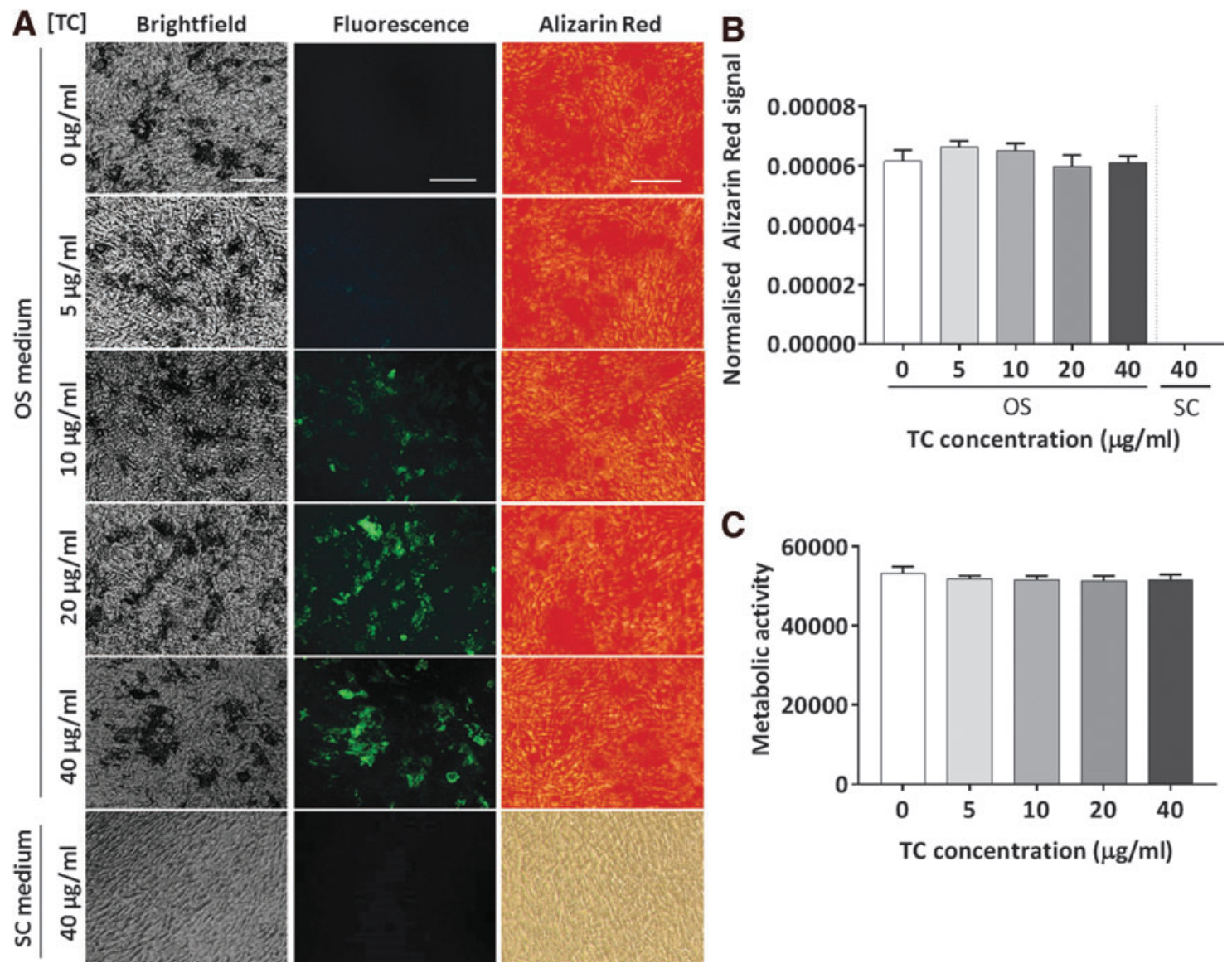

FIG. 1. Human MSC cultures exposed to increasing doses of TC at day 20 of differentiation treatment. Scale bar: $125 \mu \mathrm{m}$. (A) Representative images of cells cultured in SC or OS media after TC (green) and Alizarin Red S staining. (B, C) Quantification of Alizarin Red S staining (B) and metabolic activity $(\mathbf{C}) .(n=3)$. MSCs, mesenchymal stem cells; OS, osteogenic medium; SC, standard medium; TC, tetracycline. Color images available online at www.liebertpub.com/tec

concentrations. Live fluorescence imaging revealed a green staining pattern that colocalized with the grainy mineral deposits visible on the top of the cell monolayer in brightfield mode. The signal was stronger and more defined as the TC concentration increased, while no staining was detected in either differentiated cells unexposed to TC or in undifferentiated cells treated with $40 \mu \mathrm{g} / \mathrm{mL}$ of TC (Fig. 1A).

Alizarin Red S staining performed at day 21 in postfixed cells confirmed the differentiated phenotype of cells transiently exposed to the four concentrations of TC and showed no significant differences between all treatment groups $(p=0.47)$, confirming that TC exposure did not interfere with the staining (Fig. 1A, B). Semiquantitation of DNA amount (Supplementary Fig. S1; Supplementary Data are available online at www.liebertpub.com/tec) and analysis of metabolic activity (Fig. 1C) performed in cells treated with increasing TC concentrations also showed no differences $(p=0.77)$ among the different groups, confirming that the TC treatment was nontoxic at all concentrations tested in this study.

The fluorescence intensity observed in TC-treated cultures was measured using a microplate reader, and TC signal was significantly higher in cells treated with osteogenic condition than in undifferentiated cells maintained in SC medium (Fig. 2). The $40 \mu \mathrm{g} / \mathrm{mL}$ concentration resulted in significantly higher values in comparison to all the other concentrations in both culture conditions (Fig. 2).

\section{Tetracycline fluorescence spectrum and co-staining with other fluorescent dyes}

To define the optimal imaging settings for TC cell labelling, the lambda mode of the confocal microscope was used with $405,488,561$, or $633 \mathrm{~nm}$ lasers to detect the whole TC spectra in fixed cells treated with $20 \mu \mathrm{g} / \mathrm{mL}$ of TC. Strong emission was detected when a $405 \mathrm{~nm}$ laser was used with two major peaks at 520 and $584 \mathrm{~nm}$. Importantly, relatively low signals $(<8 \%)$ were observed in the emission spectra acquired at other wavelengths $(488,561$, and $633 \mathrm{~nm})$, suggesting little or no signal spillover (Supplementary Fig. S2).

To test the possibility of using TC concomitantly with other fluorescent dyes, fixed cells treated with $20 \mu \mathrm{g} / \mathrm{mL}$ of TC were co-stained with Hoechst 33258 and TRITCPhalloidin to label nuclei and cytoskeleton, respectively. Notably, using the $405 \mathrm{~nm}$ laser, a residual Hoechst 33258 signal was detected at $520 \mathrm{~nm}$ but not at $584 \mathrm{~nm}$. Therefore, the TC imaging protocol was optimized using the $405 \mathrm{~nm}$ laser with detection in the range of $588-650 \mathrm{~nm}$, providing a specific TC signal without any spillover signal from Hoechst 33258 or Phalloidin-TRITC fluorophores (Fig. 3). 


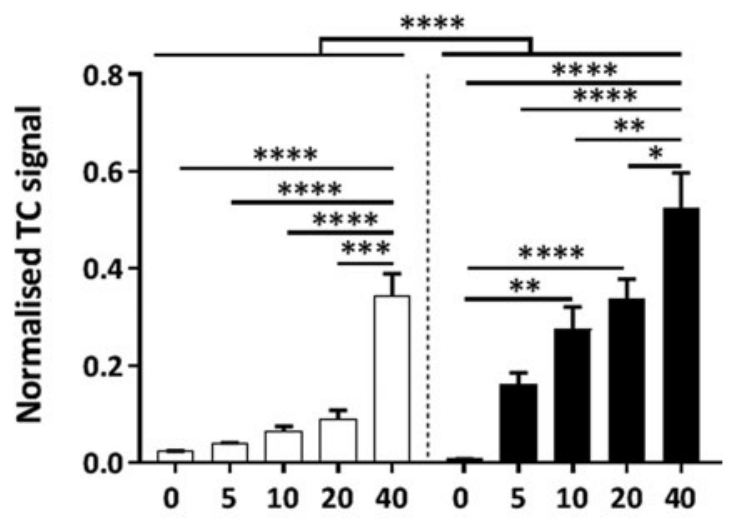

TC concentration $(\mu \mathrm{g} / \mathrm{ml})$

\section{SC medium OS medium}

FIG. 2. Quantification of TC fluorescence in MSCs cultured during 21 days in SC (white) or OS (black) medium containing increasing TC doses. SC, standard medium; OS, osteogenic medium. $* p<0.05, * * p<0.01, * * * p<0.001$, $* * * * p<0.0001$.

\section{Live Tetracycline staining in differentiating cells}

To further optimize TC staining for live monitoring of osteogenic differentiation, TC administration to live cultures was performed in either single or serial doses $(20 \mu \mathrm{g} / \mathrm{mL})$ at days 6,13 , and 20 (Fig. 4A), to evaluate the biocompatibility and efficiency of multiple TC exposures. Cells exposed to single or repeated TC treatment were first observed under fluorescence microscopy to evaluate the nature and distribution of the staining overtime (Fig. 4B). Live imaging revealed visible TC-stained mineral deposits at days 14 and 21 of OS treatment, but not at day 7 , suggesting minimal differentiation at this time point. At day 14, the TC staining pattern and signal intensity were similar in cells exposed to single and serial TC doses. At day 21 of differentiation however, a sharper and more defined signal was observed after serial administration of TC (three consecutive doses) in comparison to a single administration, suggesting a cumulative effect of serial TC treatment from day 14 but not from day 7 .

Quantification of TC fluorescence was performed in living cells at days 7,14 , and 21 of OS differentiation to complement the microscopy observation, confirming timedependent increase in TC signal (Fig. 5A). The administration of serial doses resulted in significantly higher TC fluorescence intensity at day 21 in comparison to the single dose administration protocol. Power analysis performed to evaluate the assay's sensitivity showed that the administration of TC $20 \mu \mathrm{g} / \mathrm{mL}$ had a power of $97 \%$ and $100 \%$ to discriminate twofold and fivefold differences with three samples in each group, and to discriminate a 1.5 -fold difference the assay's power was of $78 \%$ with six samples.

When analyzing cellular parameters, TC exposure was not found to negatively affect metabolic activity after either single or serial doses at any of the time points analyzed (Fig. 5B), and measurements of ALP activity at days 7 and 14 showed comparable levels in cells after single or serial TC treatment and cells unexposed to TC (Fig. 5C). The Alizarin Red S staining performed confirmed the progression of cell differentiation from days 7 to 21 (Supplementary Fig. S3). Importantly, staining quantification (Fig. 5D) revealed no significant differences in mineral deposition at any time point between untreated and TC-treated cells, whether using single or serial doses, indicating that TC treatment did not interfere with the progression of cellular differentiation $(p>0.9999)$.

To confirm the versatility of the assay, the serial administration of $20 \mu \mathrm{g} / \mathrm{mL}$ TC was also performed to human primary MSCs, which constitutes a clinical relevant cell type but also to the mouse D1 immortalized MSCs which is widely used in in vitro studies (see Supplementary Data). Live fluorescence imaging showed for both cell types a sharp and defined staining pattern at day 21 after three treatments with TC (Supplementary Fig. S4).

\section{Discussion}

The use of in vitro live cell assays permits the monitoring and evaluation of cell status in ongoing cultures, allowing longitudinal assessment of cellular responses in real time. In the field of bone research and tissue engineering this is important as in vitro studies involve long-term experiments to allow for ECM maturation and mineralization, which are typically considered over 3 weeks of cell culture. The assessment of the ALP enzymatic activity in living cells allows for the evaluation of the status of the cell differentiation in real time during the in vitro culture period; however, this targets the early stage of differentiation. ${ }^{2}$ Moreover, molecular
FIG. 3. Representative images of OS-treated cells at day 21 stained with TC $20 \mu \mathrm{g} / \mathrm{mL}$ (green) or only phosphate buffered saline and counterstained with Hoechst 33258 (blue) and Phalloidin (red). Scale bar: $125 \mu \mathrm{m}$. Color images available online at www.liebertpub .com/tec
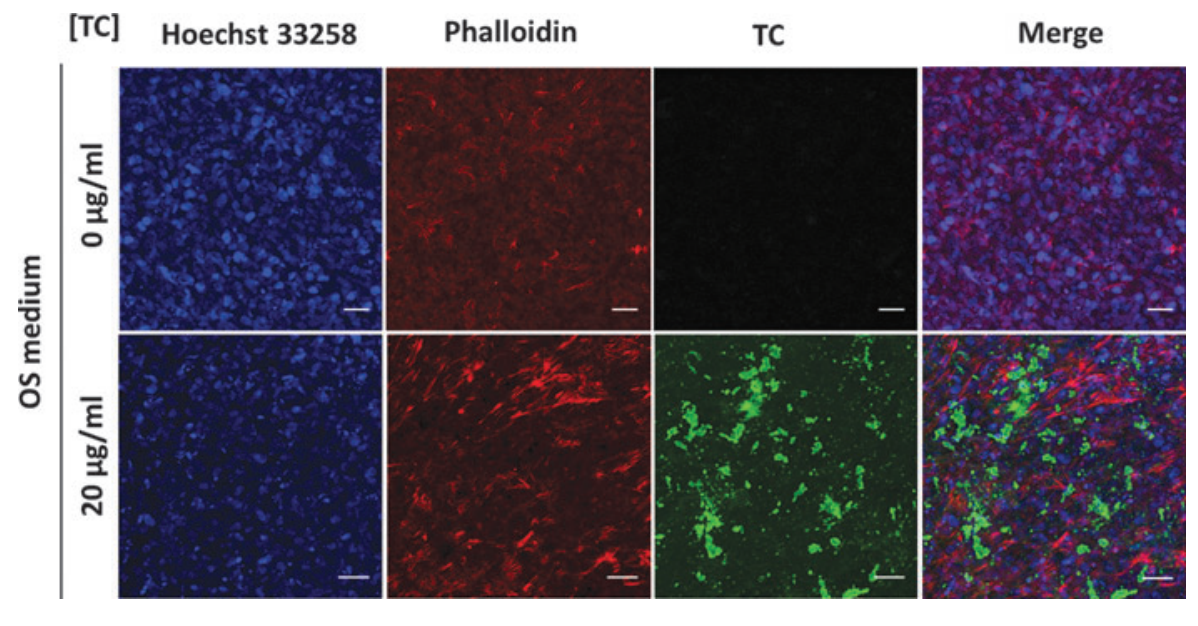
A
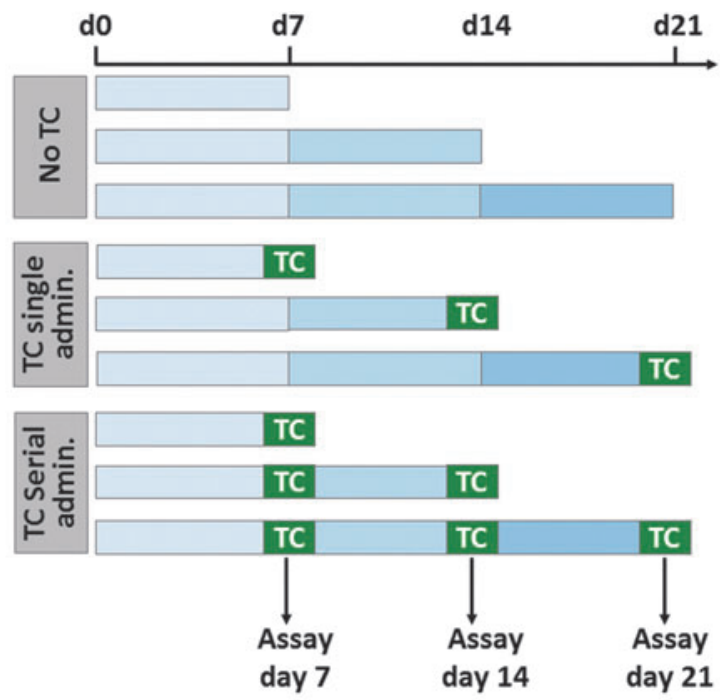

B

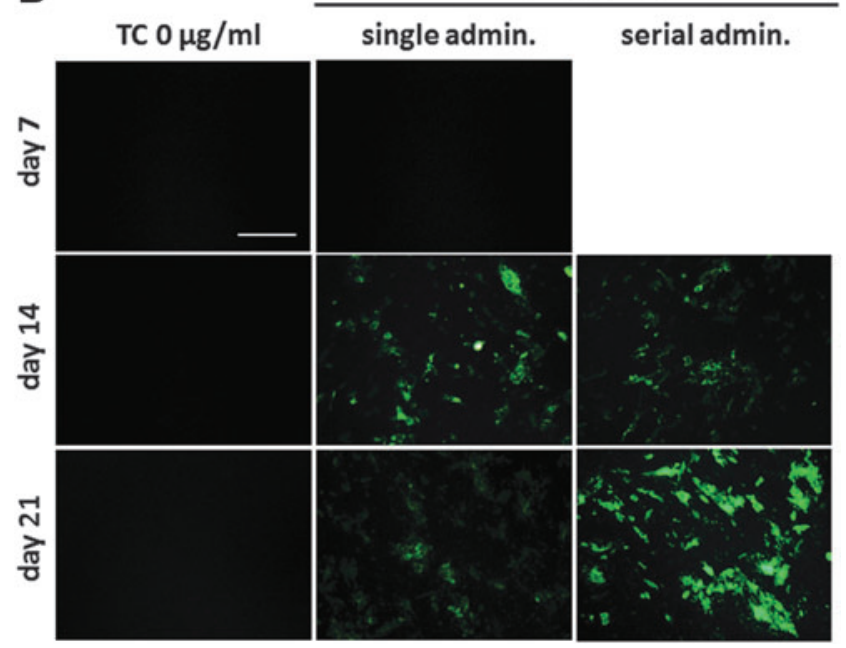

FIG. 4. Live fluorescence monitoring of MSC differentiation using single or serial doses of TC. (A) Diagram and timeline of TC administration to live cells under osteogenic treatment for 7, 14, and 21 days. (B) Representative fluorescence images of OS-treated live MSCs at days 7, 14, and 21 after single and serial TC exposure $(20 \mu \mathrm{g} / \mathrm{mL}$, green $)$ compared to unexposed controls. Scale bar: $125 \mu \mathrm{m}$. Color images available online at www.liebertpub.com/tec

reporter systems engineered using osteogenic gene promoters to drive a reporter marker can allow for a direct follow-up of gene expression and differentiation profile ${ }^{20}$; however, these imply genetic manipulations therefore preventing their direct application in primary cells. To overcome these limitations, we have developed a new protocol for the nondestructive measurement of mineralization in live cultures using tetracycline hydrochloride. We have also defined the optimal regime of TC administration to enable staining, quantification, and fluorescence imaging in live cells using human MSCs as a differentiation model. We have also showed that TC staining can be multiplexes with other fluorophores to enable advanced cellular analysis of osteogenic cultures.

Among the several tetracycline derivatives available and already in use for in vivo bone labeling, we selected tetracy- cline hydrochloride as it displayed the highest brightness in comparison to others when used to label rat bone samples. ${ }^{21}$ Our results showed that TC administrated in vitro as single or repeated doses did not alter the metabolic activity of human MSCs. The results of several studies suggest that different administration protocols and/or TC derivatives may differently affect cell health and also that sensitivity to TC derivatives might be cell type dependent. Indeed, MG-63 human osteosarcoma cells experienced a decrease in number after overnight incubation with $10 \mu \mathrm{g} / \mathrm{mL}$ doxycycline and a significant reduction of proliferation after daily treatment with the same dose of the compound. ${ }^{22}$ The treatment of primary human osteoblasts with $190 \mu \mathrm{g} / \mathrm{mL}$ of TC resulted in a $30 \%$ increase of lactate dehydrogenase, which was considered as an indication of impaired mitochondrial function, and suggested that this cytotoxic effect could be partially mediated by an alteration of mitochondrial respiration. ${ }^{23}$ Another study reported that the administration of TC analogs (doxycycline, COL-3, and minocycline) from $10 \mu \mathrm{g} / \mathrm{mL}$ to the acute myeloid leukemia cell line HL60 reduced cell viability of $>50 \%$. By contrast, $1 \mu \mathrm{g} / \mathrm{mL}$ of doxycycline and minocycline continuously added to the cell culture medium have been reported to significantly increase the proliferation of human osteoblastic bone marrow cells. ${ }^{24,25}$ Our results also showed that TC administration at days 7 and 14 during the culture period did not interfere with the induction and progression of the differentiation process. For both ALP activity and mineral deposition assays, no significant differences were observed between cells treated with single and serial doses of TC in comparison to cultures unexposed to TC. In this regard too, existing reports on the effect of TC treatment on bone differentiation are contradictory and might depend on the TC derivative used, the dose, and the administration regime. In vitro, the daily administration of $1 \mu \mathrm{g} / \mathrm{mL}$ of doxycycline or minocycline seemed to significantly promote the mineralization of human bone marrow-derived osteoblasts. ${ }^{25}$ In vivo, several studies performed on diverse disease models (such as diabetes, osteopenia, and osteoporosis) in mice and rats reported a positive association between the administration of TC derivatives and bone formation and density. ${ }^{26-29}$ However, it has been proposed that such an effect observed in vivo might be mediated by an inhibitory effect of TC on osteoclast function. $^{29,30}$ As an opposite trend, TC administration at early stages of development or to ex vivo embryonic bones, bone growth was compromised followed by increase in bone fragility. ${ }^{31,32}$

The present study also demonstrated that TC is a suitable compound for the evaluation of osteogenic differentiation in living cells such as MSCs, over an extended culture period. All the TC doses tested in this study $(5-40 \mu \mathrm{g} / \mathrm{mL})$ were selected within the same range as previously reported ${ }^{14,32}$ and were suitable for the fluorescent labeling of mineralizing cells, resulting in a more defined signal as the concentration increased. This trend was confirmed by spectrophotometric analysis, which revealed a linear increase of the detected fluorescence signal. However, among the TC doses tested in this study, $20 \mu \mathrm{g} / \mathrm{mL}$ was selected as optimum to monitor the progression of MSC differentiation at various time points. While the highest dose $(40 \mu \mathrm{g} / \mathrm{mL})$ provided a more defined and brighter signal in fluorescent images, the spectrophotometric quantitation also showed a significant increase of the signal in undifferentiated cells, suggesting the 
FIG. 5. Time course analysis of the single or serial TC exposure $(20 \mu \mathrm{g} / \mathrm{mL})$ on MSC cultures compared to no TC exposure. (A) TC fluorescence signal quantitation showing increased signal upon repeated exposure. (B-D) Cell metabolic activity (B), ALP activity (C), and Alizarin Red S staining quantitation (D) in MSC cultures showing no significant difference across all conditions. $n=3$. ALP, alkaline phosphatase.
A

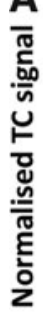

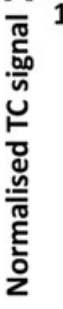

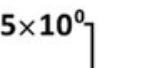

$5 \times 10^{0} 7$

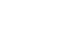

.
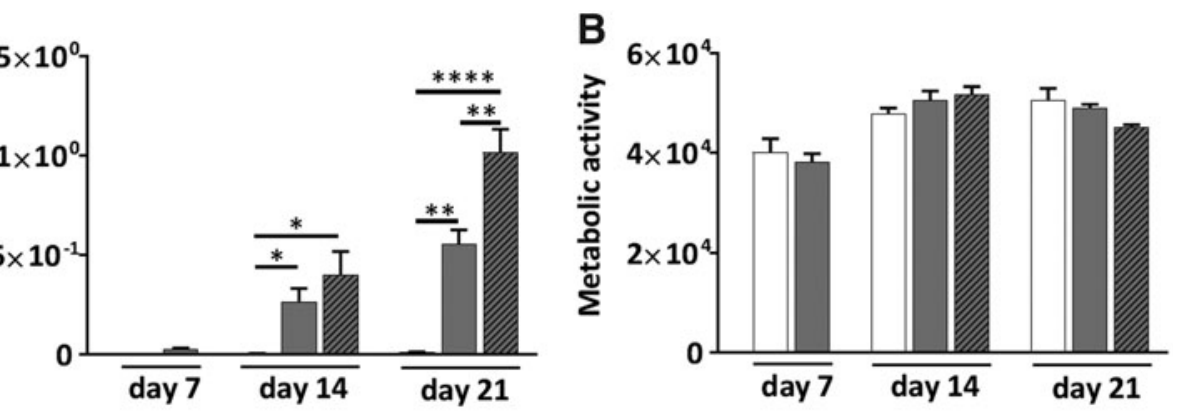

No TC $\square$ TC single admin. $\mathbb{Z}$ TC serial admin.

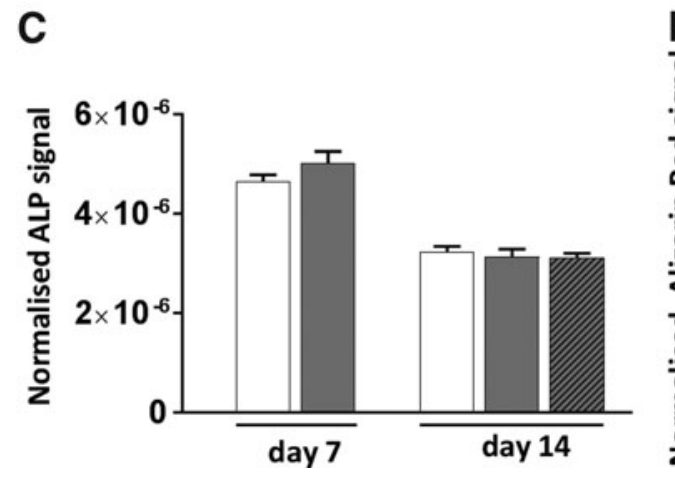

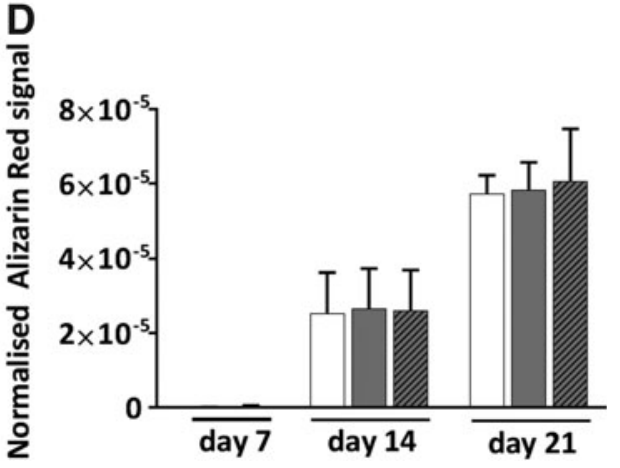

increase of unspecific background signal at this concentration. In our study, TC labeling was clearly visible as green stain using a conventional DAPI long pass filter at days 14 and 21 , but not at day 7 of osteogenic treatment. This result was in agreement with the spectrophotometric quantitation of TC fluorescence signal and was also confirmed by parallel Alizarin Red S staining, which did not reveal any detectable mineral deposits at day 7 . Due to the calcium tropism of TC, the presence of calcium phosphate minerals in the ECM is necessary for the staining. The production of mineralized ECM is a late event during bone-lineage differentiation, starting around days 10-12, while the earlier period is characterized by active cell proliferation and production of ECM components. ${ }^{2}$ Serial TC administration resulted in a stronger and more defined staining in MSC cultures, particularly at day 21 , in comparison to the single dose administration. This result, observed by microscopy and confirmed by spectrophotometry, suggests the retention and accumulation of the dye into the mineralizing bone-like nodules between the different administrations over the 21 days of culture. This appears in agreement with in vivo studies reporting the retention and visualization of this compound in bones up to 4 weeks after administration. ${ }^{33}$ These results confirm the suitability of TC staining to monitor the progression of live MSC differentiation at various time points, using a simple and cell neutral protocol.

The real-time evaluation of mineralizing cultures has previously been achieved by the continuous administration of Calcein without affecting the cell viability or the progression of differentiation, resulting in a green labeling visible through a FITC long pass filter. ${ }^{8}$ However, this method is based on the uptake of Calcein by living cells; therefore its use is limited to live cultures while the TC can also be used in fixed samples. ${ }^{3,13}$ In this regard, it is worth noting that TC allows for the multiplex staining with fluorophores visible in other channels, including the nuclear dye Hoechst 33258 and TRITC-conjugated Phalloidin as shown in our study, and is therefore a versatile tool for immunohistochemistry analysis. Moreover, our results show that the quantitative assessment of mineralization at specific time points can be achieved by an incubation of $<24 \mathrm{~h}$ with TC resulting in quantifiable and visible signal.

In summary, in this study we have developed a new protocol for the quantitative monitoring and quantitation of cell mineralization in real time based on the use of tetracycline hydrochloride. In comparison to conventional histological methods such as Alizarin Red S and Von Kossa staining, which are end point assays and require multiple steps for staining and dye quantification, the use of TC is cytocompatible, can be performed in live cells lowering the number of cells required for longitudinal studies, and is both straightforward and economical. Overnight incubation results in visible and quantifiable fluorescence signal, which can be quantified by spectrophotometry directly from live cultures while being compatible with the use of other fluorophores in live and fixed samples, enabling multiplex immunohistochemistry analysis. TC cell labeling enables the in vitro evaluation of osteogenic differentiation in different cell models, including human primary stem cells, and can thus support research into bone repair and tissue engineering targeting new pro-osteogenic approaches.

\section{Conclusions}

In this study, we have described a new nondestructive method for the live and quantitative staining of MSC cultures undergoing osteogenic differentiation, based on the transient administration of tetracycline hydrochloride during the culture period. The results showed this method to be nontoxic, sensitive, and quantitative. TC can be selectively 
imaged by fluorescent microscopy in live cells and enables the concomitant use of other fluorophores, which offers a useful and versatile method for high throughput analysis of osteogenic cultures.

\section{Acknowledgments}

This article summarizes independent research funded by the National Institute for Health Research (NIHR) under its i4i Challenge Award Programme (Grant Ref. No. II-C30714-20001). The views expressed are those of the authors and not necessarily those of the NHS, the NIHR, or the Department of Health. The authors are grateful to the Tissue Engineering group (School of Pharmacy, University of Nottingham) for the use of the microplate reader and the Leica DM IRB microscope. Confocal imaging was performed with the help of Robert Markus, School of Life Sciences Imaging (SLIM), of the University of Nottingham. This work was supported by the Biotechnology and Biological Sciences Research Council [grant number BBSRC BB/L013827/1]; and the Engineering and Physical Sciences Research Council [doctoral training centre scholarship]. The Zeiss Elyra PS.1 microscope, the processing computers and software were funded by BBSRC BB/ L013827/1.

\section{Disclosure Statement}

The authors declare that they have no conflict of interests.

\section{References}

1. Boskey, A.L., and Roy, R. Cell culture systems for studies of bone and tooth mineralization. Chem Rev 108, 4716, 2008.

2. Stein, G.S., Lian, J.B., and Owen, T.A. Relationship of cell growth to the regulation of tissue-specific gene expression during osteoblast differentiation. FASEB J 4, 3111, 1990.

3. Hayashi, O., Katsube, Y., Hirose, M., Ohgushi, H., and Ito, $\mathrm{H}$. Comparison of osteogenic ability of rat mesenchymal stem cells from bone marrow, periosteum, and adipose tissue. Calcif Tissue Int 82, 238, 2008.

4. Bills, C.E., Eisenberg, H., and Pallante, S.L. Complexes of organic acids with calcium phosphate: the Von Kossa stain as a clue to the composition of bone mineral. Johns Hopkins Med J 128, 194, 1974.

5. Puchtler, H., Meloan, S.N., and Terry, M.S. On the history and mechanism of Alizarin and Alizarin Red S stains for calcium. J Histochem Cytochem 17, 110, 1969.

6. Wang, Y.H., Liu, Y., Maye, P., and Rowe, D.W. Examination of mineralized nodule formation in living osteoblastic cultures using fluorescent dyes. Biotechnol Prog 22, 1697, 2006.

7. van Gaalen, S.M., Kruyt, M.C., Geuze, R.E., de Bruijn, J.D., Alblas, J., and Dhert, W.J. Use of fluorochrome labels in in vivo bone tissue engineering research. Tissue Eng Part B Rev 16, 209, 2010.

8. Uchimura, E., Machida, H., Kotobuki, N., et al. In-situ visualization and quantification of mineralization of cultured osteogenetic cells. Calcif Tissue Int 73, 575, 2003.

9. Querido, W., Farina, M., and Balduino, A. Giemsa as a fluorescent dye for mineralizing bone-like nodules in vitro. Biomed Mater 7, 011001, 2012.
10. Golub, L.M., Suomalainen, K., and Sorsa, T. Host modulation with tetracyclines and their chemically modified analogues. Curr Opin Dent 2, 80, 1992.

11. Pautke, C., Vogt, S., Kreutzer, K., et al. Characterization of eight different tetracyclines: advances in fluorescence bone labeling. J Anat 217, 76, 2010.

12. Finerman, G.A., and Milch, R.A. In vitro binding of tetracyclines to calcium. Nature 198, 486, 1963.

13. McClure, J. Demonstration of calcification fronts by in vivo and in vitro tetracycline labelling. J Clin Pathol 35, 1278, 1982.

14. Wang, X., Jin, T., Chang, S., et al. In vitro differentiation and mineralization of dental pulp stem cells on enamellike fluorapatite surfaces. Tissue Eng Part C Methods 18, 821, 2012.

15. Okamoto, T., Aoyama, T., Nakayama, T., et al. Clonal heterogeneity in differentiation potential of immortalized human mesenchymal stem cells. Biochem Biophys Res Commun 295, 354, 2002.

16. France, L.A., Scotchford, C.A., Grant, D.M., Rashidi, H., Popov, A.A., and Sottile, V. Transient serum exposure regimes to support dual differentiation of human mesenchymal stem cells. J Tissue Eng Regen Med 8, 652, 2014.

17. Harrison, R., Markides, H., Morris, R.H., Richards, P., El Haj, A.J., and Sottile, V. Autonomous magnetic labelling of functional mesenchymal stem cells for improved traceability and spatial control in cell therapy applications. J Tissue Eng Regen Med 11, 2333, 2016.

18. Rashidi, H., Strohbuecker, S., Jackson, L., et al. Differences in the pattern and regulation of mineral deposition in human cell lines of osteogenic and non-osteogenic origin. Cells Tissues Organs 195, 484, 2012.

19. Sottile, V., Halleux, C., Bassilana, F., Keller, H., and Seuwen, K. Stem cell characteristics of human trabecular bone-derived cells. Bone 30, 699, 2002.

20. Born, A.K., Lischer, S., and Maniura-Weber, K. Watching osteogenesis: life monitoring of osteogenic differentiation using an osteocalcin reporter. J Cell Biochem 113, 313, 2012.

21. Pautke, C., Schieker, M., Tischer, T., et al. Characterization of osteosarcoma cell lines MG-63, Saos-2 and U-2 OS in comparison to human osteoblasts. Anticancer Res 24, 3743, 2004.

22. Fife, R.S., Rougraff, B.T., Proctor, C., and Sledge, G.W., Jr. Inhibition of proliferation and induction of apoptosis by doxycycline in cultured human osteosarcoma cells. J Lab Clin Med 130, 530, 1997.

23. Duewelhenke, N., Krut, O., and Eysel, P. Influence on mitochondria and cytotoxicity of different antibiotics administered in high concentrations on primary human osteoblasts and cell lines. Antimicrob Agents Chemother 51, 54, 2007.

24. Song, H., Fares, M., Maguire, K.R., Siden, A., and Potacova, Z. Cytotoxic effects of tetracycline analogues (doxycycline, minocycline and COL-3) in acute myeloid leukemia HL-60 cells. PLoS One 9, e114457, 2014.

25. Gomes, P.S., and Fernandes, M.H. Effect of therapeutic levels of doxycycline and minocycline in the proliferation and differentiation of human bone marrow osteoblastic cells. Arch Oral Biol 52, 251, 2007.

26. Sasaki, T., Kaneko, H., Ramamurthy, N.S., and Golub, L.M. Tetracycline administration restores osteoblast struc- 
ture and function during experimental diabetes. Anat Rec 231, 25, 1991.

27. Bain, S., Ramamurthy, N.S., Impeduglia, T., Scolman, S., Golub, L.M., and Rubin, C. Tetracycline prevents cancellous bone loss and maintains near-normal rates of bone formation in streptozotocin diabetic rats. Bone 21, 147, 1997.

28. Williams, S., Barnes, J., Wakisaka, A., Ogasa, H., and Liang, C.T. Treatment of osteoporosis with MMP inhibitors. Ann N Y Acad Sci 878, 191, 1999.

29. Williams, S., Wakisaka, A., Zeng, Q.Q., et al. Effect of minocycline on osteoporosis. Adv Dent Res 12, 71, 1998.

30. Zhou, X., Zhang, P., Zhang, C., An, B., and Zhu, Z. Tetracyclines inhibit rat osteoclast formation and activity in vitro and affect bone turnover in young rats in vivo. Calcif Tissue Int 86, 163, 2010.

31. Engesaeter, L.B., Lie, S.A., Espehaug, B., Furnes, O., Vollset, S.E., and Havelin, L.I. Antibiotic prophylaxis in total hip arthroplasty: effects of antibiotic prophylaxis systemically and in bone cement on the revision rate of 22,170 primary hip replacements followed 0-14 years in the Norwegian Arthroplasty Register. Acta Orthop Scand 74, 644, 2003.
32. Kaitila, I., Wartiovaara, J., Laitinen, O., and Saxen, L. The inhibitory effect of tetracycline on osteogenesis in organ culture. J Embryol Exp Morphol 23, 185, 1970.

33. Buyske, D.A., Eisner, H.J., and Kelly, R.G. Concentration and persistence of tetracycline and chlortetracycline in bone. J Pharmacol Exp Ther 130, 150, 1960.

Address correspondence to:

Virginie Sottile, PhD

Wolfson STEM Centre

Division of Cancer and Stem Cells

School of Medicine

The University of Nottingham

CBS building, University Park

Nottingham NG7 2RD

United Kingdom

E-mail: virginie.sottile@nottingham.ac.uk

Received: September 19, 2017

Accepted: January 4, 2018

Online Publication Date: February 27, 2018 